\title{
DcR3 promotes hepatoma cell migration by downregulating E-cadherin expression
}

\author{
HONGLING ZHANG ${ }^{1 *}, \mathrm{XUHONG} \mathrm{CHEN}^{2 *}, \mathrm{DONGMING} \mathrm{LI}^{3}, \mathrm{LULU}^{2} \mathrm{CUI}^{1}$, \\ XIN LI ${ }^{1}$, XIUFENG $\mathrm{YE}^{2}$ and XIAOCHUN WAN ${ }^{1}$ \\ ${ }^{1}$ Shenzhen Laboratory of Fully Human Antibody Engineering, Institute of Biomedicine and Biotechnology, \\ Shenzhen Institutes of Advanced Technology, Chinese Academy of Sciences, Shenzhen 518055; \\ ${ }^{2}$ Shenzhen Second People's Hospital, The First Affiliated Hospital of Shenzhen University, Shenzhen 518035; \\ ${ }^{3}$ Basic Medicine College, Guangxi Medical University, Nanning, Guangxi 530021, P.R. China
}

Received November 23, 2016; Accepted December 27, 2016

DOI: 10.3892/or.2017.5685

\begin{abstract}
Decoy receptor 3 (DcR3), a decoy molecule belonging to the tumor necrosis factor receptor superfamily (TNFRSF), is a soluble receptor that can neutralize the biological effects of three other TNFSF members, namely, Fas ligand (FasL/TNFSF6/CD95L), LIGHT (TNFSF14) and TNF-like molecule 1A (TL1A/TNFSF15). DcR3 expression is increased in tumor cells. As such, DcR3 has been considered a potential biomarker to predict cancer invasion and progression of inflammation. However, the molecular mechanisms of DcR3 in tumor progression and metastasis remain poorly described. In the present study, DcR3 induced cytoskeleton remodeling, inhibited E-cadherin expression, and promoted cancer cell migration. Immunofluorescence and flow cytometry demonstrated that DcR3 expression was increased in hepatoma cells, whereas E-cadherin expression was significantly downregulated. Immunohistochemistry revealed that DcR3 and E-cadherin exhibited an opposite expression pattern between normal and cancerous liver tissues. Moreover, DcR3 treatment promoted $\mathrm{I} \kappa \mathrm{B} \alpha$ degradation and p65 nuclear translocation. Therefore, the present study uncovered the mechanism underlying the function of DcR3 in cancer cell migration and provides evidence that DcR3 may be a potential target for cancer therapy.
\end{abstract}

Correspondence to: Dr Xiaochun Wan, Shenzhen Laboratory of Fully Human Antibody Engineering, Institute of Biomedicine and Biotechnology, Shenzhen Institutes of Advanced Technology, Chinese Academy of Sciences, Shenzhen 518055, P.R. China

E-mail: xc.wan@siat.ac.cn

Dr Xiufeng Ye, Shenzhen Second People's Hospital, The First Affiliated Hospital of Shenzhen University, Shenzhen 518035, P.R. China

E-mail: szrosexiu@126.com

${ }^{*}$ Contributed equally

Key words: DcR3, cell migration, E-cadherin

\section{Introduction}

Decoy receptor 3 (DcR3), a soluble molecule belonging to the tumor necrosis factor receptor superfamily (TNFRSF), was first identified as a decoy receptor of Fas ligand (FasL) and inhibitor of FasL-induced apoptosis (1). DcR3 also neutralizes the biological effects of two other TNFSF members, namely, LIGHT (TNFSF14) and TNF-like molecule 1A (TL1A/TNFSF15) (2-4). DcR3 can be defined as an immunomodulator on the basis of its neutralizing effects on FasL, LIGHT and TL1A (4-7). DcR3 is upregulated in tumor cells and inflammatory diseases (8-11). DcR3 in serum can be used as a biomarker to predict cancer invasion and progression of inflammation (12-15). DcR3 also acts as an effector molecule to modulate cell function through non-decoy activities, including the effects on cell adhesion and differentiation (16-18).

Liver cancer is a common malignancy worldwide. Cancerous liver tissues yield high DcR3 expression, and this expression is correlated with tumor differentiation, serosal invasion and liver metastases $(13,19,20)$. Nevertheless, the precise mechanisms of DcR3 in liver cancer progression and metastasis remain unclear.

E-cadherin, a classical member of the cadherin superfamily, is a calcium-dependent cell-cell adhesion glycoprotein (21). The E-cadherin-catenin complex plays a key role in cellular adhesion $(22,23)$. The loss of E-cadherin function or expression has been implicated in cancer progression and metastasis $(24,25)$. In the present study, DcR3 treatment caused HepG2 cell cytoskeleton remodeling, inhibited E-cadherin expression, and promoted cell migration. Immunohistochemical analysis revealed that E-cadherin and DcR3 exhibited an opposite expression trend in liver carcinoma tissues. The present study also demonstrated the functional mechanism of DcR3 in cancer cell migration and provides a theoretical basis for the use of a DcR3 antagonist to treat liver cancer.

\section{Materials and methods}

Clinical samples. Tissue samples from three patients with hepatic carcinoma and biliary tract disease were collected during surgical resection performed at the Shenzhen Second 
People's Hospital (Shenzhen, China). Liver tumor and non-tumor liver tissues were fixed and immediately used to prepare tissue slices. All of the samples were obtained with patient consent and approval of the Institutional Animal Care and Use Committee, Shenzhen Institutes of Advanced Technology.

Cell culture and transfection. The human hepatocarcinoma cell line HepG2 or the normal liver cell line L02 were obtained from the Shanghai Institute of Cell Biology (Shanghai, China). Both cell lines were maintained in Dulbecco's modified Eagle's medium (DMEM), supplemented with $10 \%$ fetal bovine serum (FBS) (Gibco, Carlsbad, CA, USA), and $100 \mu \mathrm{g} / \mathrm{ml}$ each of penicillin-streptomycin (HyClone, Logan, UT, USA) in 5\% $\mathrm{CO}_{2}$ at $37^{\circ} \mathrm{C}$.

In 6-well plates, $2 \times 10^{5}$ cells/well were cultured overnight and transfected with $2 \mu \mathrm{g}$ PLVX-IRES-ZsGreen-DcR3 plasmid with Lipofectamine ${ }^{\circledR} 2000$ (Invitrogen, Carlsbad, CA, USA). The cells transfected with an empty vector were used as a blank control.

Western blot analysis. HepG2 cells were treated with $3 \mu \mathrm{g} / \mathrm{ml}$ DcR3-Fc or IgG1 as control, or transfected with the indicated plasmids. At $48 \mathrm{~h}$ after transfection or treatment, cells were harvested and determined by the antibodies indicated in the figures. Cell pellets were lysed in RIPA (Thermo Fisher Scientific Inc., Waltham, MA, USA), $1 \mathrm{mM}$ phenylmethylsulfonyl fluoride (PMSF) (Beyotime, Shanghai, China) and 1\% protease inhibitor cocktail (Thermo Fisher Scientific, Inc.). Lysates were normalized for total protein $(25 \mu \mathrm{g})$ and loaded on $8-12 \%$ sodium dodecyl sulfate-polyacrylamide gel, electrophoresed, and transferred to a polyvinylidene fluoride (PVDF) membrane (Millipore, Kenilworth, NJ, USA), followed by blocking with $5 \%$ skimmed milk at room temperature for $1 \mathrm{~h}$. The membrane was incubated with primary antibodies overnight at $4^{\circ} \mathrm{C}$, and rinsed with Tris-buffered saline with Tween-20. The blots were then incubated with horseradish peroxidase (HRP)-conjugated secondary antibody (KPL) for $1 \mathrm{~h}$ at room temperature. Detection was performed using EMD Millipore Luminata $^{\mathrm{TM}}$ Western HRP Chemiluminescence Substrates (WBLUR0500). Nuclear and cytoplasmic extracts were isolated with NE-PER ${ }^{\text {TM }}$ Nuclear and Cytoplasmic Extraction Reagents (78833) purchased from Thermo Fisher Scientific. The defined sections of the film were scanned for image capture and quantification using Adobe Photoshop software (CS4; (Adobe Systems, Inc., San Jose, CA, USA) and ImageJ software (National Institutes of Health, Bethesda, MD, USA).

$R N A$ isolation and real-time quantitative PCR. Total RNA was isolated using TRIzol reagent (Invitrogen) according to the manufacturer's instructions. RNA samples were reverse transcribed with oligo (dT) and M-MLV Reverse Transcriptase (Takara, Tokyo, Japan). A mixture of $1 \mu \mathrm{g}$ RNA, $4 \mu \mathrm{l}$ 5X RT mix, $1 \mu 1$ primer mix, and nuclease-free water were made up to a 20- $\mu 1$ volume. The reverse transcription step was as follow: $37^{\circ} \mathrm{C}$ for $15 \mathrm{~min} ; 85^{\circ} \mathrm{C}$ for $5 \mathrm{sec}$, and then stored at $-20^{\circ} \mathrm{C}$. Realtime quantitative PCR analysis was performed with specific primers for human E-cadherin (forward, 5'-TGGAGGAATTC TTGCTTTGC-3' and reverse, 5'-CGTACATGTCAGCCAGC TTC-3') in a CFX96 Touch $^{\mathrm{TM}}$ Real-Time PCR Detection
System (Bio-Rad, Hercules, CA, USA) with SYBR qPCR mix (Takara). Relative levels of gene expression were determined using GAPDH as the control (forward, 5'-ATCTGGCACCAC ACCTTCTAC-3' and reverse, 5'-CAGCCAGGTCCAGACGC AGG-3'). SYBR-Green PCR Master Mix $2 \mu \mathrm{l}$, forward and reverse primers $200 \mathrm{nM}$, cDNA template $100 \mathrm{ng}$, and $\mathrm{ddH}_{2} \mathrm{O}$ up to $10 \mu \mathrm{l}$ volume was mixed together. PCR conditions consisted of the following: $95^{\circ} \mathrm{C}$ for 3 min for denaturation; $95^{\circ} \mathrm{C}$ for $5 \mathrm{sec}$ for annealing; and $60^{\circ} \mathrm{C}$ for $40 \mathrm{sec}$ for extension, for 40 cycles. The threshold cycle for each sample was selected from the linear range and converted to a starting quantity by interpolation from a standard curve generated on the same plate for each set of primers. The E-cadherin mRNA levels were normalized for each well to the GAPDH mRNA levels using the $2^{-\Delta \Delta \mathrm{Ct}}$ method.

Immunofluorescent assay. L02 or HepG2 cells were washed with phosphate-buffered saline (PBS) and fixed at room temperature with $4 \%$ polyformaldehyde for $10 \mathrm{~min}$, permeated with $0.1 \%$ Triton X-100 for 7 min, blocked for $30 \mathrm{~min}$ with $1 \%$ $\mathrm{BSA}$, and incubated sequentially with the indicated primary and secondary antibodies. 4',6-Diamidino-2-phenylindole (DAPI) (Beyotime) was used to label the nuclei. PhalloidinRhodamine (Thermo Fisher Scientific) was used for F-actin staining.

Flow cytometry. L02 or HepG2 cells were collected and washed with PBS, fixed and permeabilized with Fix/Perm solution (BioLegend, San Diego, CA, USA) before intracellular staining. After $15 \mathrm{~min}$, the cells were washed twice with Perm/Wash buffer, and incubated with the DcR3 antibody at $4^{\circ} \mathrm{C}$ for $1 \mathrm{~h}$. Cells were washed with PBS and incubated with the FITC-goat anti-rabbit antibody at $4^{\circ} \mathrm{C}$ for $30 \mathrm{~min}$. Cells were washed with PBS twice. The intracellular fluorescence of FITC was detected by FCM after excitation at $488 \mathrm{~nm}$. Fluorescence emissions at $530 \mathrm{~nm}$ from 10,000 cells were collected, amplified and scaled to generate a single-parameter histogram.

Immunochemistry. The sample sections were deparaffinized and rehydrated. After boiling in a microwave oven, the antigen was retrieved with a $0.01 \mathrm{M}$ sodium citrate buffer $(\mathrm{pH}$ 6.0) at a sub-boiling temperature for $20 \mathrm{~min}$. The following steps were performed with the SP kit (9001; ZSGB-BIO, Beijing, China). Shortly, the sections were incubated with $3 \%$ hydrogen peroxide for $10 \mathrm{~min}$ to block endogenous peroxidase. After $15 \mathrm{~min}$ of pre-incubation in 5\% normal goat serum to prevent non-specific staining, the samples were incubated with the antibody to DcR3 (Abcam, Cambridge, UK) at $4^{\circ} \mathrm{C}$ overnight. Secondary antibody was added and incubated for $30 \mathrm{~min}$. The sections were incubated in horseradish enzyme-labeled chain avidin solution for $30 \mathrm{~min}$ at room temperature. Color was developed with a diaminobenzidine (DAB) substrate kit. Counterstaining was performed with hematoxylin.

Wound healing assay. Confluent cell cultures were grown on 6-well plates. Wounds were made with the tip of a micropipette. DcR3-Fc was added to the culture medium at a final concentration of $3 \mu \mathrm{g} / \mathrm{ml}$. IgG1 was added as a control. Wound closure speed was analyzed as indicated in the legend. 


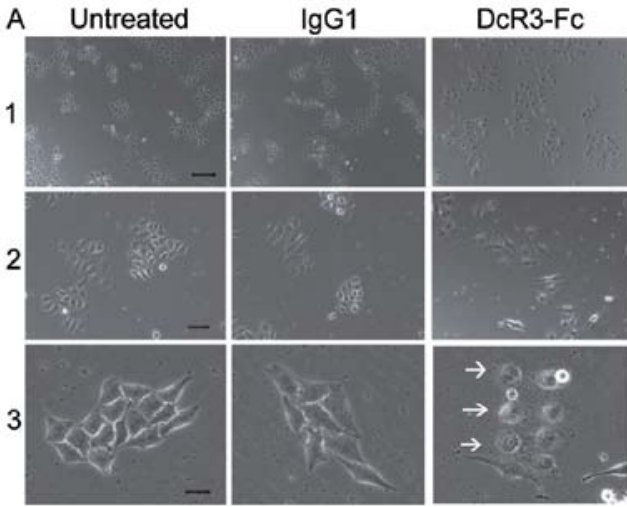

C

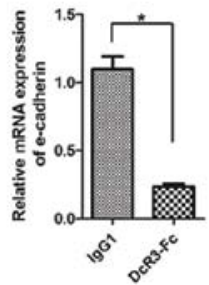

B
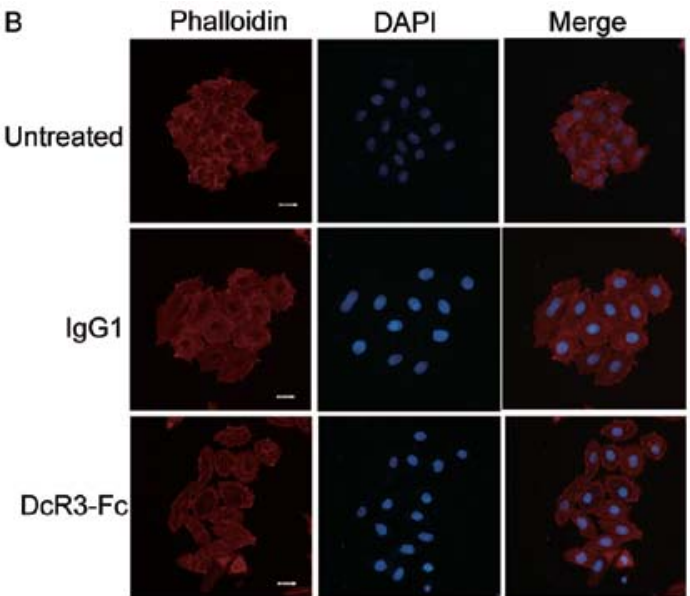

$\mathrm{E}$
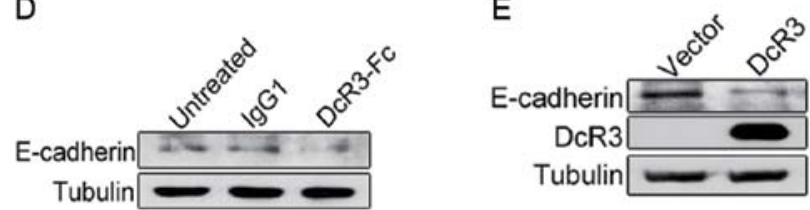

Figure 1. DcR3 regulates colony scattering and inhibits E-cadherin expression. Cells were plated at a low density and treated with $3 \mu \mathrm{g} / \mathrm{ml}$ DcR3-Fc or control IgG1. The colony morphology was analyzed 4 days after plating. (A) Representative images of colony formation. Rows 1-3 indicate colony scattering under different magnification. Scale bar: row 1,150 $\mu \mathrm{m}$; row 2, $50 \mu \mathrm{m}$ and row 3,20 $\mu \mathrm{m}$. (B) Representative images of actin remodeling as observed by phalloidin staining. Scale bar, $20 \mu \mathrm{m}$. (C) HepG2 cells were treated with $3 \mu \mathrm{g} / \mathrm{ml}$ DcR3-Fc. Forty-eight hours later, the E-cadherin mRNA level was determined by qRT-PCR. Data are from 3 indepentdent experiments; * $\mathrm{p}<0.05$. (D) HepG2 cells were treated as described in C, and the protein level of E-cadherin was detected using western blot analysis. Representative images from two independent experiments are shown. (E) HepG2 cells were transfected with the control or DcR3-expressing vectors. At $48 \mathrm{~h}$ after transfection, cells were harvested and whole-cell lysates were detected by the indicated antibodies. Representative images from two independent experiments are shown.

Transwell assay. HepG2 cells were treated with $3 \mu \mathrm{g} / \mathrm{ml}$ DcR3-Fc or IgG for $24 \mathrm{~h}$, and then were trypsinized and resuspended in DMEM without FBS before plating on the upper layer of the Transwell with an $8-\mu \mathrm{m}$ pore-size membrane at a cell density of $1 \times 10^{4}$. DMEM containing $5 \%$ FBS was added to the lower layer. After $15 \mathrm{~h}$, the cells remaining on the top surface were scratched off. The cells on the lower surface were fixed in methanol, stained with $0.5 \%$ crystal violet (Beyotime), and images were captured under a microscope. The intact Transwell was dissolved in 33\% acetic acid, and the supernatant was detected for absorption values with a spectrophotometer at $590 \mathrm{~nm}$.

Materials. DcR3-Fc and human IgG1 proteins were purchased from Sino Biological, Inc. (Beijing, China). Anti-DcR3, anti-E-cadherin and anti-I $\mathrm{B} \alpha$ antibodies were purchased from Abcam. Anti-p65 was purchased from Santa Cruz Biotechnology (Santa Cruz, CA, USA). Anti-tubulin was purchased from Abmart (Shanghai, China).

Statistical analysis. All experiments were repeated at least three times or noted otherwise. Data are expressed as mean \pm SD. The t-test was performed for inter-group comparisons. Values with $\mathrm{p}<0.05$ were considered to show significant differences.

\section{Results}

$D c R 3$ regulates colony scattering of Hep $G 2$ cells and decreases $E$-cadherin expression. HepG2 cells were examined using a colony scattering assay to analyze the function of DcR3 in regulating cell migration. The colony scattering assay, mimicking certain aspects of tumor invasion, reveals the ability of epithelial tumor cells to detach from colonies in culture. The cells were plated at very low density, and the morphological characteristics of the colonies were evaluated 5 days after plating. The colonies were compact in the control or IgG1 groups, and $>90 \%$ of the cells in a colony contained cell-cell junctions. By contrast, the cells were scattered in the DcR3 treatment group, and $<20 \%$ of the cells formed junctions (Fig. 1A). In the cells detached from the scattered colonies, numerous protrusions were formed on the membrane edge of these cells (Fig. 1A, lane 3, arrows). Philloidin staining revealed that DcR3 promoted actin remodeling and revealed a scattered phenotype (Fig. 1B). This finding indicated that DcR3 triggered changes in cell morphology and enhanced the ability of cells to detach from the colonies.

DcR3 treatment disrupts colony scattering and causes cytoskeleton remodeling in HepG2 cells. To investigate the role of DcR3 in the regulation of cell-cell adhesion, we detected whether E-cadherin, a key molecule in the regulation of intercellular adhesion, was regulated by DcR3. The mRNA of E-cadherin was significantly downregulated by DcR3 treatment (Fig. 1C). The same effect was observed at the protein level (Fig. 1D). DcR3 expression also inhibited E-cadherin expression (Fig. 1E). Thus, DcR3 is a negative regulator of E-cadherin.

$D c R 3$ and E-cadherin expression levels are inversely correlated in hepatocarcinoma cell lines and tissues. To understand 
A
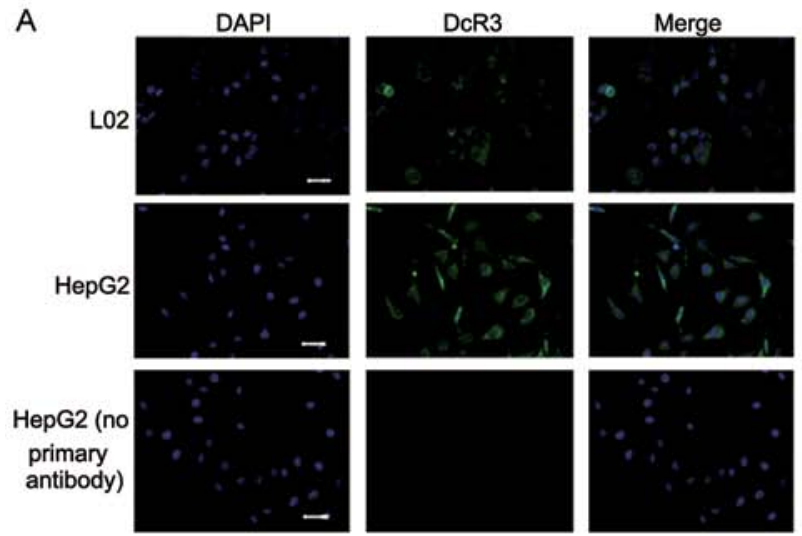

E

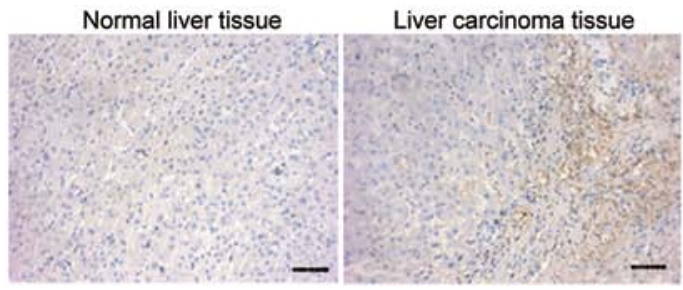

B
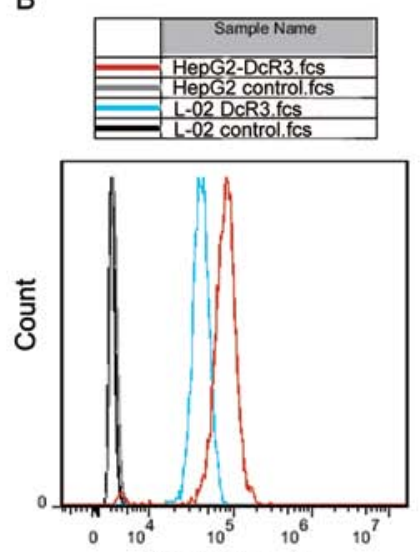

FL1-H::FL1-H

F

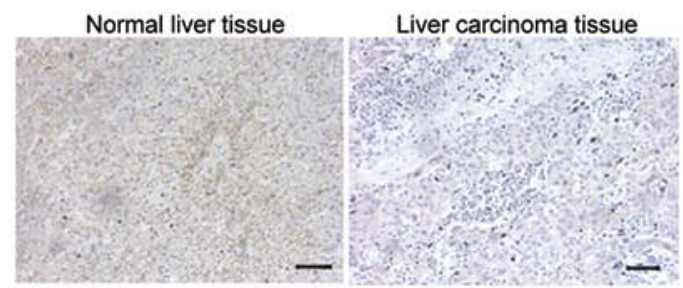

C

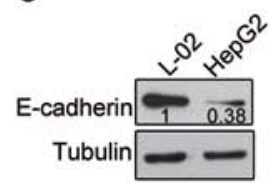

D

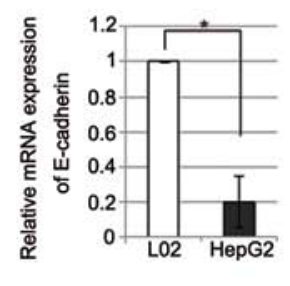

iver carcinoma tissue

Figure 2. DcR3 and E-cadherin expression in hepatocarcinoma cell lines and tissues. DcR3 expression in L02 and HepG2 cells was determined using (A) immunofluorescence assay (scale bar, $50 \mu \mathrm{m}$ ) and (B) flow cytometry. E-cadherin protein or mRNA expression in L02 and HepG2 cells was determined using (C) western blot analysis and (D) qRT-PCR; ${ }^{p}$ < $<0.05$. Representative images of (E) DcR3 expression in normal and liver carcinoma tissues and (F) E-cadherin. Scale bar, $200 \mu \mathrm{m}$.

A

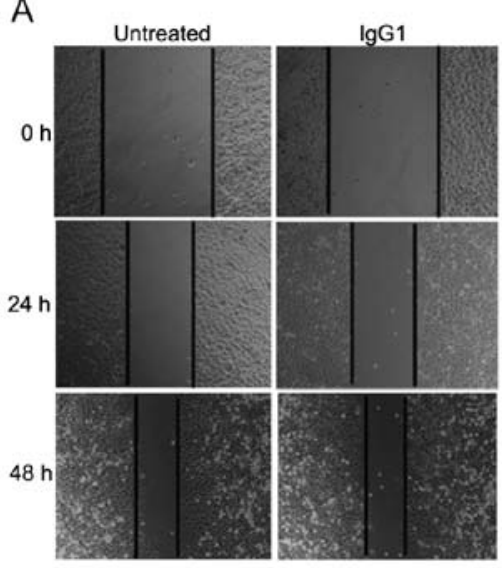

C

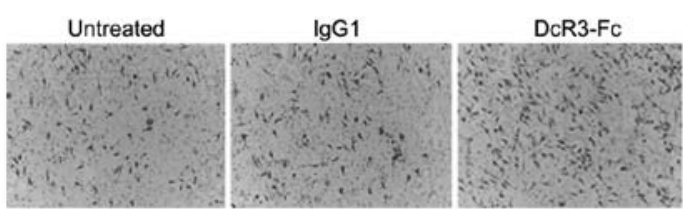

B
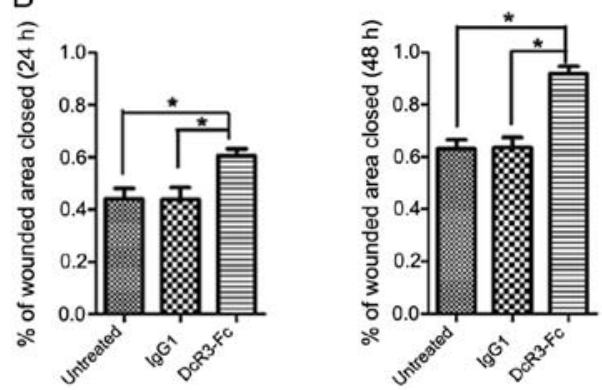

$\mathrm{D}$

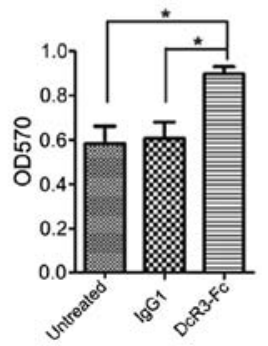

$\mathrm{E}$

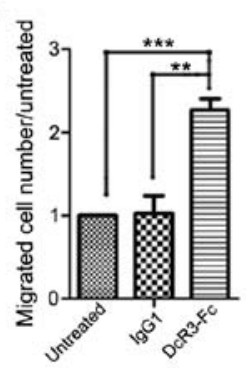

Figure 3. DcR3 treatment promotes cell migration. (A) Representative images of wound healing assay as described in Materials and methods. (B) Quantitative analysis of three independent experiments as presented in A; ${ }^{\mathrm{p}}<0.05$. (C) Representative images of the Transwell assay as described in the Materials and methods. (D and E) Quantitative analysis of three independent experiments as presented in $\mathrm{C} ;{ }^{*} \mathrm{p}<0.05,{ }^{* *} \mathrm{p}<0.01,{ }^{* * *} \mathrm{p}<0.001$.

the significance of the role of DcR3 in hepatocarcinoma, we detected DcR3 and E-cadherin expression levels in the normal liver cell line L02 and the hepatoma cell line HepG2 by performing immunofluorescent assays. The DcR3 expression level was higher in the HepG2 cells than that noted in the L02 cells (Fig. 2A). To confirm this observation, we detected the 
A

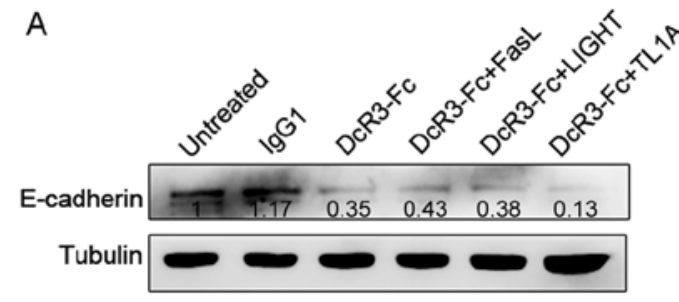

B

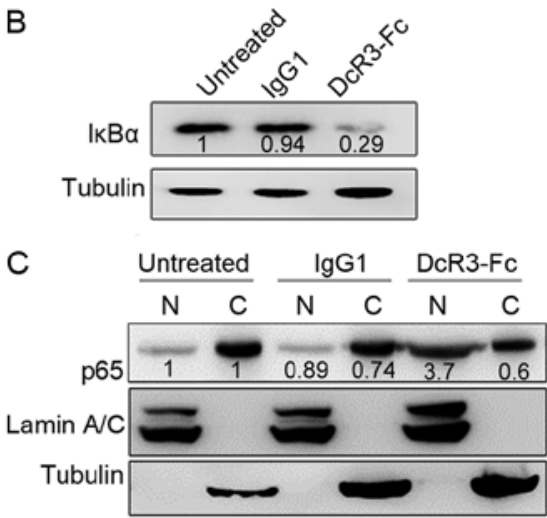

D

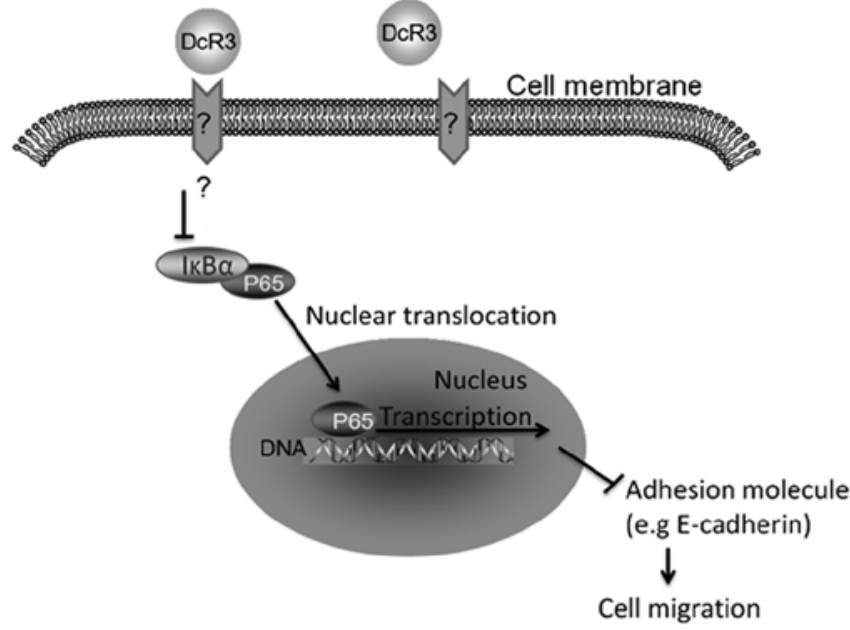

Figure 4. DcR3 deregulates I $\mathrm{B} \alpha$ expression and induces p65 nuclear translocation. (A) HepG2 cells were treated with $3 \mu \mathrm{g} / \mathrm{ml}$ control IgG1 or $3 \mu \mathrm{g} / \mathrm{ml} \mathrm{DcR} 3-\mathrm{Fc}$ alone or with $3 \mu \mathrm{g} / \mathrm{ml}$ FasL, LIGHT and TL1A. After $48 \mathrm{~h}$, cells were harvested and E-cadherin expression was detected by western blot analysis. (B) HepG2 cells were treated with $3 \mu \mathrm{g} / \mathrm{ml}$ control IgG1 or $3 \mu \mathrm{g} / \mathrm{ml}$ DcR3-Fc. I $\kappa \mathrm{B} \alpha$ expression was detected by western blot analysis at $48 \mathrm{~h}$ after treatment. (C) HepG2 cells were treated as described in B, p65 expression in the cytoplasm and nucleus was determined by western blot analysis. p65 in the cytoplasm (C) or the nucleus (N) were normalized to lamin A/C or tubulin, respectively. Representative images from 2 independent experiments are shown. (D) Schematic illustration of the role of DcR3 in regulating cell migration.

DcR3 expression using flow cytometry. The DcR3 expression level in HepG2 cells was higher than that in the L-02 cells (Fig. 2B). Protein and mRNA quantification showed that E-cadherin was downregulated in the HepG2 cells as compared with that in the L-02 cells (Fig. 2C and D). Importantly, immunohistochemical staining showed that DcR3 was almost undetectable in the non-tumor liver tissues (from patients with biliary tract disease) but was upregulated in the liver cancer tissues (Fig. 2E). Inversely, E-cadherin was located in the cell junction in non-tumor tissue, but was almost undetectable in liver cancer tissue (Fig. 2F). Therefore, hepatocarcinomas exhibited low E-cadherin expression but high DcR3 levels.

DcR3 promotes cancer cell migration. Considering that E-cadherin plays a crucial role in cell adhesion and migration, we analyzed the effect of DcR3 on the migratory ability of HepG 2 cells. The wound healing assay demonstrated that the addition of the DcR3-containing supernatant caused a strong increase in cell migration at 24 and $48 \mathrm{~h}$ (Fig. 3A and B). To confirm this trend, we detected the cell migratory ability using a Transwell assay. We observed that DcR3 greatly enhanced HepG2 cell migration (Fig. 3C-E). These results also indicated that DcR3 plays a positive role in cancer cell migration.

DcR3 induces p65 cytoplasm-nuclear translocation. To investigate whether DcR3 inhibits E-cadherin expression via its decoy function, we treated HepG 2 cells by simultaneously adding DcR3 and its ligand FasL, LIGHT or TL1A, to the culture medium. The addition of these ligands did not affect the function of DcR3 in the regulation of E-cadherin expression (Fig. 4A). Thus, DcR3 inhibited E-cadherin expression via its non-decoy function.
To investigate the mechanism of the function of DcR3 in E-cadherin regulation, we explored the involved signaling pathway. DcR3 regulates the $\mathrm{NF} \kappa \mathrm{B}$ signaling pathway in monocytes. E-cadherin is also a target of p65 $(26,27)$. Consequently, we investigated whether DcR3 activates NFкB signaling in HepG2 cells. DcR3 significantly downregulated IkB $\alpha$ expression (Fig. 4B). Furthermore, DcR3 treatment of HepG2 cells markedly increased the nuclear translocation of the NF- $\kappa$ B subunit p65 (Fig. 4C). These data suggest that the $N F \kappa B$ signaling cascade is an essential component in the involvement of E-cadherin expression for the DcR3-mediated migration response.

\section{Discussion}

DcR3 can be defined as a novel immunosuppressant on the basis of its neutralizing effects on FasL, LIGHT and TL1A. DcR3 is expressed by tumor cells from various lineages, including adenocarcinomas of the colon, rectum $(28,29)$, lung (30) and gastric cancer $(31)$, hepatocellular carcinoma $(13,20)$ and in chronic liver diseases (19), which frequently lead to cancer formation. Increased DcR3 levels in serum or tissues were found to be correlated with poor prognosis and resistance to treatment in some cancer patients (13). In addition to its neutralizing effect, DcR3 also acts as an effector molecule to modulate cell function via non-decoy activities, including the regulation of DC and macrophage differentiation that leads to Th2 polarization $(32,33)$, M2 macrophage differentiation (34), and cytoskeleton remodeling $(16,17)$.

DcR3 can induce actin reorganization in human monocytes, and this protein triggers multiple signaling molecules, such as PKC and phosphatidylinositol 3-kinase (PI3K) (35). 
Furthermore, DcR3 induces NFkB-mediated expression of ICAM-1, VCAM-1 and IL-8 by monocytes; consequently, their binding to endothelial cells is enhanced (17). DcR3-Fc was found to act on THP-1 monocytes and differentiated macrophages to increase the expression level of integrin $\alpha 4$. Thus, cell aggregation and proliferation are promoted and apoptosis is reduced (18). DcR3 is upregulated in cancer cells; thus, these observations suggest its important roles in modulating the migration and trafficking of monocytes/macrophages in the tumor microenvironment.

E-cadherin plays an important role in cell adhesion by forming adherent junctions to bind cells within tissues. The loss of E-cadherin expression has been defined as a hallmark of EMT. EMT is a process by which epithelial cells lose their cell polarity and cell-cell adhesion, and they gain migratory and invasive properties to become mesenchymal stem cells. DcR3 was found to induce I $\kappa$ B kinase activation, I $\kappa$ B degradation, and p65 nuclear translocation in human microvascular endothelial cells (17). E-cadherin is a target of p65, which represses E-cadherin expression and enhances the epithelialto-mesenchymal transition of mammary epithelial cells via ZEB-1 and ZEB-2 (36). In addition, TGF- $\beta$ is one of the most critical factors involved in EMT regulation. TGF- $\beta$ promotes EMT through both Smad-dependent and Smad-independent manner (37). The relationship between DcR3 and the TGF- $\beta$ pathway will be the research of interest in a future study. A model of the function of DcR3 in cell migration regulation is shown in Fig. 4D. DcR3 controls the expression of E-cadherin and the p65 translocation of HepG2 cells. Thus, it elicits double effects in tumor metastasis regulation and immune modulation. The blocking of DcR3 may be applied as an effective therapeutic strategy to prevent tumor metastasis.

In conclusion, the present study demonstrated that DcR3 is overexpressed in hepatic carcinoma tissues and cell lines. DcR3-Fc treatment inhibited E-cadherin expression, enhanced tumor cell migration in vitro, and promoted p65 nuclear translocation. These findings revealed the mechanism underlying the ability of DcR3 to regulate cell migration. Therefore, DcR3 may be a potential target for the gene therapy of hepatic carcinoma.

\section{Acknowledgements}

The present study was supported by the Shenzhen Basic Research Program (JCYJ20150630114942293, JCYJ20140416180228582 and JCYJ20160229201353324), the Nature Science Foundation of China for Young Scholar grant (81501356), the Shenzhen Peacock Next-generation Monoclonal Antibody Drug Research and Development Program (1110140040347265), the Fourth Group of Talents in Guangdong Province (2014-1), the Shenzhen Engineering Laboratory (2014-1677), and the Shenzhen Technology Study program (JSGG20160229202150023).

\section{References}

1. Pitti RM, Marsters SA, Lawrence DA, Roy M, Kischkel FC Dowd P, Huang A, Donahue CJ, Sherwood SW, Baldwin DT, et al: Genomic amplification of a decoy receptor for Fas ligand in lung and colon cancer. Nature 396: 699-703, 1998.
2. Yu KY, Kwon B, Ni J, Zhai Y, Ebner R and Kwon BS: A newly identified member of tumor necrosis factor receptor superfamily (TR6) suppresses LIGHT-mediated apoptosis. J Biol Chem 274: 13733-13736, 1999.

3. Migone TS, Zhang J, Luo X, Zhuang L, Chen C, Hu B, Hong JS, Perry JW, Chen SF, Zhou JX, et al: TL1A is a TNF-like ligand for DR3 and TR6/DcR3 and functions as a T cell costimulator. Immunity 16: 479-492, 2002.

4. Gill RM and Hunt JS: Soluble receptor (DcR3) and cellular inhibitor of apoptosis-2 (cIAP-2) protect human cytotrophoblast cells against LIGHT-mediated apoptosis. Am J Pathol 165: 309-317, 2004.

5. Lin WW and Hsieh SL: Decoy receptor 3: A pleiotropic immunomodulator and biomarker for inflammatory diseases, autoimmune diseases and cancer. Biochem Pharmacol 81: 838-847, 2011.

6. Wan X, Shi G, Semenuk M, Zhang J and Wu J: DcR3/TR6 modulates immune cell interactions. J Cell Biochem 89: 603-612, 2003.

7. Siakavellas SI, Sfikakis PP and Bamias G: The TL1A/DR3/DcR3 pathway in autoimmune rheumatic diseases. Semin Arthritis Rheum 45: 1-8, 2015.

8. Wu Y, Han B, Sheng H, Lin M, Moore PA, Zhang J and Wu J: Clinical significance of detecting elevated serum DcR3/TR6/M68 in malignant tumor patients. Int J Cancer 105: 724-732, 2003.

9. Otsuki T, Tomokuni A, Sakaguchi H, Aikoh T, Matsuki T, Isozaki Y, Hyodoh F, Ueki H, Kusaka M, Kita S, et al: Overexpression of the decoy receptor 3 (DcR3) gene in peripheral blood mononuclear cells (PBMC) derived from silicosis patients. Clin Exp Immunol 119: 323-327, 2000.

10. Bai C, Connolly B, Metzker ML, Hilliard CA, Liu X, Sandig V, Soderman A, Galloway SM, Liu Q, Austin CP, et al: Overexpression of M68/DcR3 in human gastrointestinal tract tumors independent of gene amplification and its location in a four-gene cluster. Proc Natl Acad Sci USA 97: 1230-1235, 2000.

11. Ge Z, Sanders AJ, Ye L, Wang Y and Jiang WG: Expression of death decoy receptor-3 (DcR3) in human breast cancer and its functional effects on breast cancer cells in vitro. J Exp Ther Oncol 9: 109-118, 2011.

12. Tu HF, Liu CJ, Liu SY, Chen YP, Yu EH, Lin SC and Chang KW: Serum decoy receptor 3 level: A predictive marker for nodal metastasis and survival among oral cavity cancer patients. Head Neck 33: 396-402, 2011.

13. Bamias G, Gizis M, Delladetsima I, Laoudi E, Siakavellas SI, Koutsounas I, Kaltsa G, Vlachogiannakos J, Vafiadis-Zouboulis I, Daikos GL, et al: Elevated serum levels of the antiapoptotic protein decoy-receptor 3 are associated with advanced liver disease. Can J Gastroenterol Hepatol 2016: 2637010, 2016.

14. Bamias G, Kaltsa G, Siakavellas SI, Papaxoinis K, Zampeli E, Michopoulos S, Zouboulis-Vafiadis I and Ladas SD: High intestinal and systemic levels of decoy receptor 3 (DcR3) and its ligand TL1A in active ulcerative colitis. Clin Immunol 137: 242-249, 2010.

15. Ao R, Du YQ, Wang Y, Chen YS and Wang BY: MMP-2 and DcR3 expression in esophageal cancer tissue and correlation with patient survival. Int J Clin Exp Med 6: 700-705, 2013.

16. Hsu MJ, Lin WW, Tsao WC, Chang YC, Hsu TL, Chiu AW, Chio CC and Hsieh SL: Enhanced adhesion of monocytes via reverse signaling triggered by decoy receptor 3. Exp Cell Res 292: 241-251, 2004.

17. Yang CR, Hsieh SL, Ho FM and Lin WW: Decoy receptor 3 increases monocyte adhesion to endothelial cells via NF-kappa B-dependent up-regulation of intercellular adhesion molecule-1, VCAM-1, and IL-8 expression. J Immunol 174: 1647-1656, 2005.

18. Tateishi K, Miura Y, Hayashi S, Takahashi M and Kurosaka M: DcR3 protects THP-1 macrophages from apoptosis by increasing integrin alpha4. Biochem Biophys Res Commun 389: 593-598, 2009.

19. Kim S, Kotoula V, Hytiroglou P, Zardavas D and Zhang L: Significance of increased expression of decoy receptor 3 in chronic liver disease. Dig Liver Dis 41: 591-598, 2009.

20. Chen G and Luo D: Expression of decoy receptor 3 in liver tissue microarrays. Natl Med J India 21: 275-278, 2008.

21. Nagar B, Overduin M, Ikura M and Rini JM: Structural basis of calcium-induced E-cadherin rigidification and dimerization. Nature 380: 360-364, 1996.

22. Benham-Pyle BW, Pruitt BL and Nelson WJ: Cell adhesion. Mechanical strain induces E-cadherin-dependent Yap1 and $\beta$-catenin activation to drive cell cycle entry. Science 348 : 1024-1027, 2015. 
23. Ishiyama N, Lee SH, Liu S, Li GY, Smith MJ, Reichardt LF and Ikura M: Dynamic and static interactions between p120 catenin and E-cadherin regulate the stability of cell-cell adhesion. Cell 141: 117-128, 2010.

24. Perl AK, Wilgenbus P, Dahl U, Semb H and Christofori G: A causal role for E-cadherin in the transition from adenoma to carcinoma. Nature 392: 190-193, 1998.

25. Nagafuchi A, Shirayoshi Y, Okazaki K, Yasuda K and Takeichi M: Transformation of cell adhesion properties by exogenously introduced E-cadherin cDNA. Nature 329: 341-343, 1987.

26. Paul A, Danley M, Saha B, Tawfik O and Paul S: PKC $\zeta$ promotes breast cancer invasion by regulating expression of E-cadherin and Zonula Occludens-1 (ZO-1) via NFKB-p65. Sci Rep 5: 12520, 2015.

27. Hayden MS and Ghosh S: Shared principles in NF-kappaB signaling. Cell 132: 344-362, 2008.

28. Yu W, Xu YC, Tao Y, He P, Li Y, Wu T, Zhu YP, Li J, Wu JX and Dai J: DcR3 regulates the growth and metastatic potential of SW480 colon cancer cells. Oncol Rep 30: 2741-2748, 2013.

29. Mild G, Bachmann F, Boulay JL, Glatz K, Laffer U, Lowy A, Metzger U, Reuter J, Terracciano L, Herrmann R, et al: DCR3 locus is a predictive marker for 5-fluorouracil-based adjuvant chemotherapy in colorectal cancer. Int J Cancer 102: 254-257, 2002.

30. Zhang Y, Luo J, He R, Huang W, Li Z, Li P, Dang Y, Chen G and Li S: Expression and clinicopathological implication of DcR3 in lung cancer tissues: A tissue microarray study with 365 cases. Onco Targets Ther 9: 4959-4968, 2016.
31. Zheng XF, Miao LY, Li S and Wang YB: Promotive effects of Dcr3 gene on the occurrence and progression of gastric cancer and its mechanism. Hepatogastroenterology 61: 880-884, 2014

32. Hsu TL, Chang YC, Chen SJ, Liu YJ, Chiu AW, Chio CC, Chen L and Hsieh SL: Modulation of dendritic cell differentiation and maturation by decoy receptor 3. J Immunol 168: 4846-4853, 2002.

33. Wu SF, Liu TM, Lin YC, Sytwu HK, Juan HF, Chen ST, Shen KL, Hsi SC and Hsieh SL: Immunomodulatory effect of decoy receptor 3 on the differentiation and function of bone marrow-derived dendritic cells in nonobese diabetic mice: From regulatory mechanism to clinical implication. J Leukoc Biol 75: 293-306, 2004

34. Chang YC, Hsu TL, Lin HH, Chio CC, Chiu AW, Chen NJ, Lin CH and Hsieh SL: Modulation of macrophage differentiation and activation by decoy receptor 3. J Leukoc Biol 75: 486-494, 2004.

35. Weissinger D, Tagscherer KE, Macher-Göppinger S, Haferkamp A, Wagener N and Roth W: The soluble Decoy Receptor 3 is regulated by a PI3K-dependent mechanism and promotes migration and invasion in renal cell carcinoma. Mol Cancer 12: 120, 2013.

36. Chua HL, Bhat-Nakshatri P, Clare SE, Morimiya A, Badve S and Nakshatri H: NF-kappaB represses E-cadherin expression and enhances epithelial to mesenchymal transition of mammary epithelial cells: Potential involvement of ZEB-1 and ZEB-2. Oncogene 26: 711-724, 2007.

37. Xu J, Lamouille S and Derynck R: TGF-beta-induced epithelial to mesenchymal transition. Cell Res 19: 156-172, 2009. 